\title{
Evaluation of the Microanatomy of the Liver via a Rapid Sample Preparation Protocol and a Table-Top Scanning Electron Microscope
}

\author{
Filip Braet
}

Australian Centre for Microscopy \& Microanalysis (ACMM), The University of Sydney, NSW 2006, Australia

\begin{abstract}
In this paper an alternative preparation and imaging method is presented that allows investigation of the fine structure of hepatic tissue within one hour after the initial fixation step. This approach involves, besides traditional fixation with glutaraldehyde, chemical drying and subsequent investigation of the samples with the new generation of user-friendly desktop scanning electron microscopes. The data presented herein reveal comparative preservation of liver ultrastructure to those that are prepared using classical sample preparation protocols. This low-cost approach permits the swift study of liver tissue subjected to various testing conditions and hence bridges the time gap between experiment and subsequent ultrastructural observation at the nanoscale.
\end{abstract}

Keywords: Electron microscopy, liver, microanatomy, hepatic morphology, ultrastructure.

\section{INTRODUCTION}

Nowadays the quest is on for alternative and quick sample preparation protocols to serve the demands of the impatient researcher and or to present a weight against the booming availability of fast cryo-sample preparation methods for electron microscopic investigation [1]. However, fast tracking classical sample preparation methods often result in the presence of structural artefacts which make an accurate assessment on the ultrastructure of the sample difficult [2]. This often leads to misleading morphological information, and hence conclusions $[3,4]$.

In this contribution an alternative quick approach is presented that allows investigation of the fine structure of hepatic tissue within one hour after the initial glutaraldehyde-fixation step. Subsequent steps involve the immediate dehydration and air drying of the sample by evaporation of hexamethyldisilazane $[5,6]$, which was immediately followed by sample mounting and sputter coating of the tissue blocks with a thin layer of gold [7]. This sample preparation method was combined with the newgeneration of table-top scanning electron microscopes. These instruments are typically characterised by their ease of use and are within the budget of every research laboratory that endeavors to explore the microanatomy of tissues and cells over micro- to nanometer scales.

The data presented herein reveal comparative preservation of ultrastructure to those that are prepared using the well-described classical combined glutaraldehydeosmium fixation method $[8,9]$, which is typically followed by time-consuming dehydration and subsequent critical point drying procedures. Furthermore, the method presented in this paper result in quality electron microscopic data at low and

*Address correspondence to this author at the Australian Centre for Microscopy and Microanalysis (ACMM), Madsen Building F09, The University of Sydney, NSW 2006, Australia; Tel: + 6129351 7619; Fax: + 6129351 7682; E-mail: filip.braet@sydney.edu.au intermediate magnifications. This magnification range is often sufficient to address the majority of the research quests.

\section{MATERIALS \& METHODS}

Tissue Preparation. The liver of eight weeks old male Sprague Dawley rats $(\mathrm{n}=4)$ was perfuse-fixed at physiological pressure $\left(12 \quad \mathrm{~cm} \quad \mathrm{H}_{2} 0\right)$ with $1.5 \%$ glutaraldehyde in $0.1 \mathrm{M} \mathrm{Na-cacodylate} \mathrm{buffer} \mathrm{with} 0.1 \mathrm{M}$ sucrose for $2 \mathrm{~min}$ at room temperature. See the paper by Wisse \& Braet et al. [10] for full experimental details regarding the perfusion-fixation procedure. Next, the liver was cut into small pieces of $1 \times 1 \times 1 \mathrm{~mm}$ with the aid of razor blade under $0.1 \mathrm{M} \mathrm{Na}$-cacodylate buffer supplemented with 0.1 $M$ sucrose, in order to allow full immersion with the dehydration vehicle (see next step). The samples were next rinsed five times for 2 min each in hexamethyldisilazane followed by hexamethyldisilazane immersion for $10 \mathrm{~min}$ [7]. Subsequently, the tissue blocks were transferred to a dessicator and allowed to air-dry for $10 \mathrm{~min}$. After drying, samples were immediately broken in half with a pair of tweezers, mounted on SEM stubs using conductive carbon tape and sputter-coated with $10 \mathrm{~nm}$ gold [7]. The different preparation steps are schematically depicted under Fig. (1).

Scanning Electron Microscopy. Samples were investigated in eucentric position at $15 \mathrm{kV}$ using the TM1000 Hitachi Tabletop Microscope (Hitachi Technologies, Japan). Digital images were captured using the auto image adjustment function running under Hitachi TM-1000 software (v1.01) interface. High-resolution data without digital zoom function were recorded at $1,280 \times 1,040 \mathrm{dpi}$ and at a working distance between 5.3 and 6.7 micrometers.

\section{RESULTS \& DISCUSSION}

Following the steps as outlined under Fig. (1), allowed the observation of the microanatomy of liver tissue within an hour after the start of the perfusion-fixation process. Examination of the tissue blocks at low magnification (Fig. 2A) resulted in the overall observation of the histological 


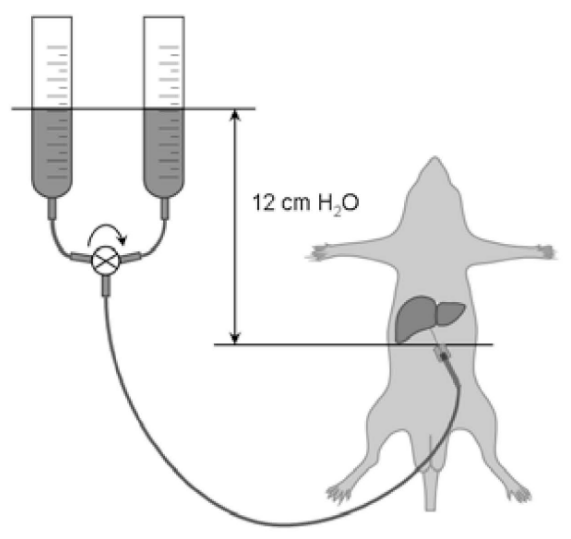

Step I (2-10 min)
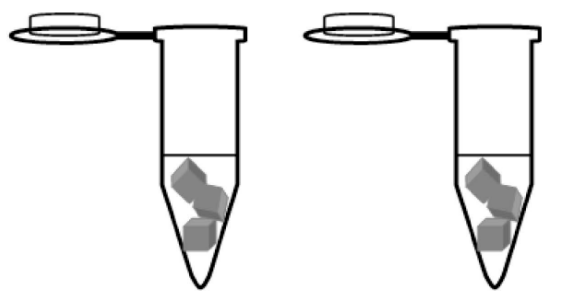

\section{Step III $(5 \times 2 \min \& 10 \mathrm{~min})$}

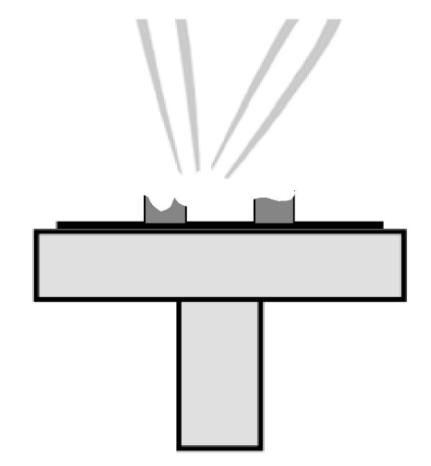

\section{Step V (10 $\min )$}

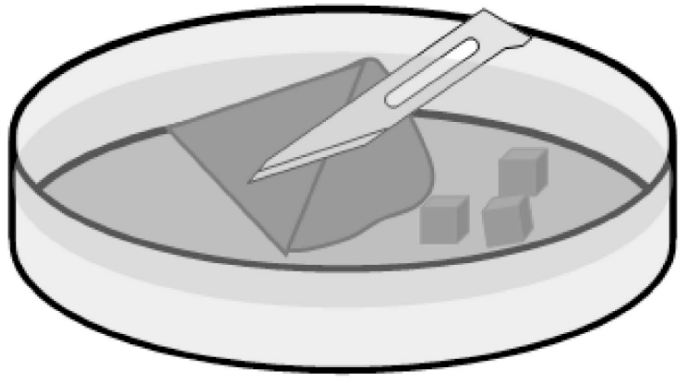

\section{Step II (10 min)}

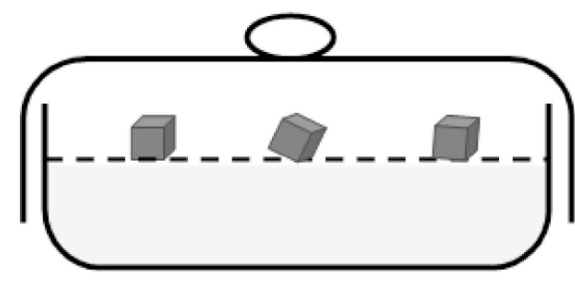

Step IV (10 min)

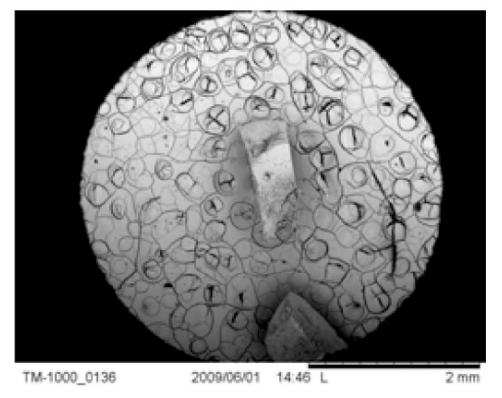

Step VI

Fig. (1). Scheme depicting the different steps involved in the preparation of liver tissue for ultrastructural scanning electron microscopy investigation. Step I, perfusion-fixation of the liver with 1.5\% glutaraldehyde; Step II, tissue slicing in blocks of $1 \mathrm{~mm}^{3}$; Step III, submerging tissue blocks in an organic reagent with a fast evaporation rate; Step IV, air-drying in a desiccator; Step V, mounting of the samples on stubs and sputter-coating; and Step VI, imaging within a table-top scanning electron microscope.

architecture of the functional liver unit [11]. Large portal areas containing branches of the vena porta, hepatic artery and bile duct could be easily descerned together with the central veins that collect the blood that flow through the microvascular bed of the liver (i.e., the liver sinusoids). At intermediate magnification, the individual sinusoids were readily visible and were surrounded by the liver parenchymal tissue that was composed of hepatocytes (Fig. 2B). Also, fine extracellular matrix material such as fine collagen bundles could be easily noted.

High-magnification imaging of the areas around the microvascular bed permitted the observation of the individual hepatocytes that contained numerous microvillous protrusions. Furthermore, bile canaliculi could be easily seen running between adjacent hepatocytes (Fig. 3A). The table- 

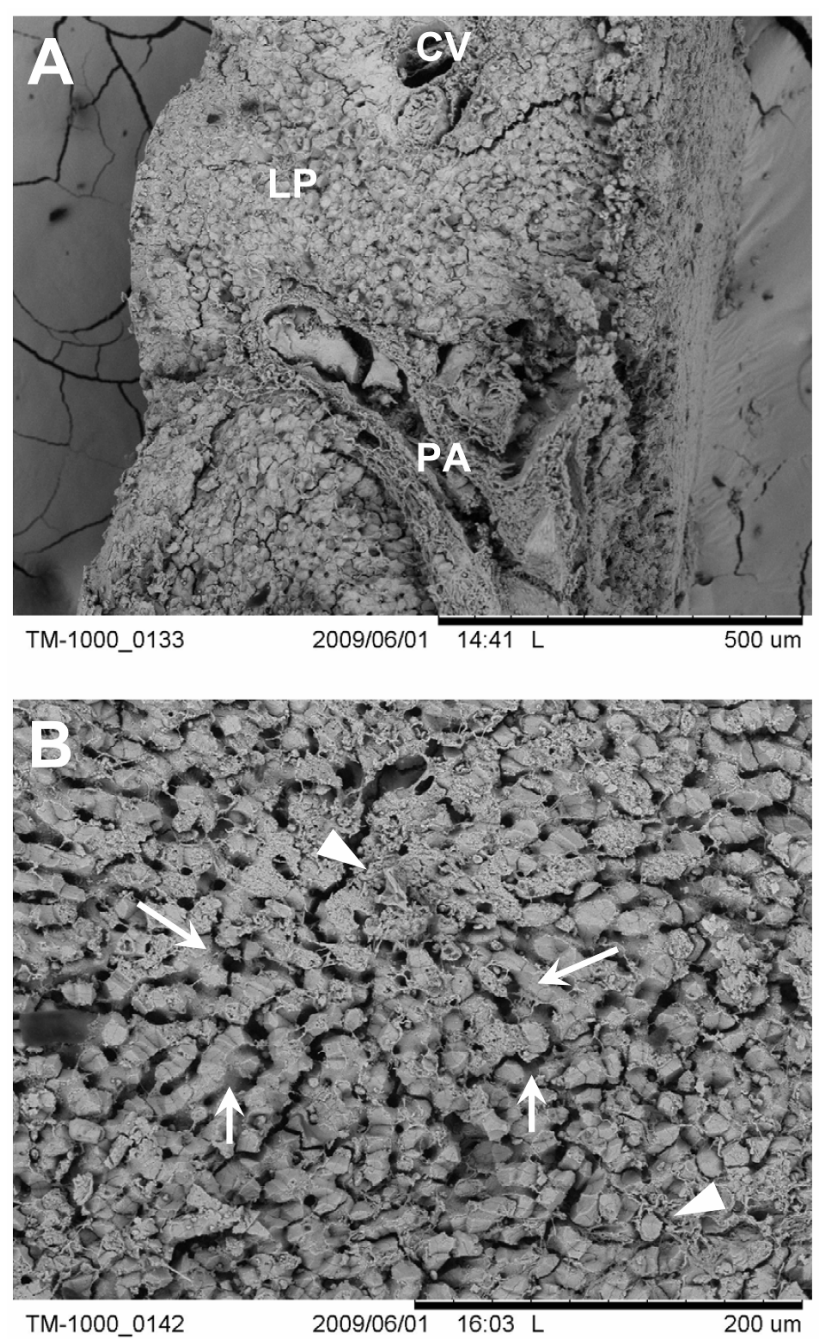

Fig. (2). Low (A) and intermediate (B) magnification of liver tissue. (A) PA, portal area; CV, central vein; LP, liver parenchyma. (B) Large arrows denote the hepatocytes that make-up the liver parenchyma and the small arrows indicate the microvascular bed or sinusoids. Arrowheads, extracellular matrix components.

top scanning electron microscopes allow magnification of samples up to 7,500-10,000 times. At this magnification range, the sinusoids disclosed their fenestrations, which have a typical size in the order of 120-160 nm (Fig. 3B). The dimension of this unique ultrastructural feature directly demonstrates the magnification capabilities of these types of table-top scanning electron microscopes.

The presence of fenestrae is also a direct structural indicator that the preparation method herein outlined is apparently sufficient enough for their preservation. This comes not as a surprise, since brief glutaraldehyde-fixation combined with cryo-fixation allowed the direct visualization of this fragile structures [12]. Noteworthy, fenestrae are known to be difficult to preserve and often collapse and/or fuse as a result of improper fixation or drying procedures $[13,14]$. In line, drying of fenestrated liver endothelial cells via hexamethyldisilazane was found to be successful in the preservation of these fragile membrane-bound pores under in vitro conditions [7]. And drying by evaporation of

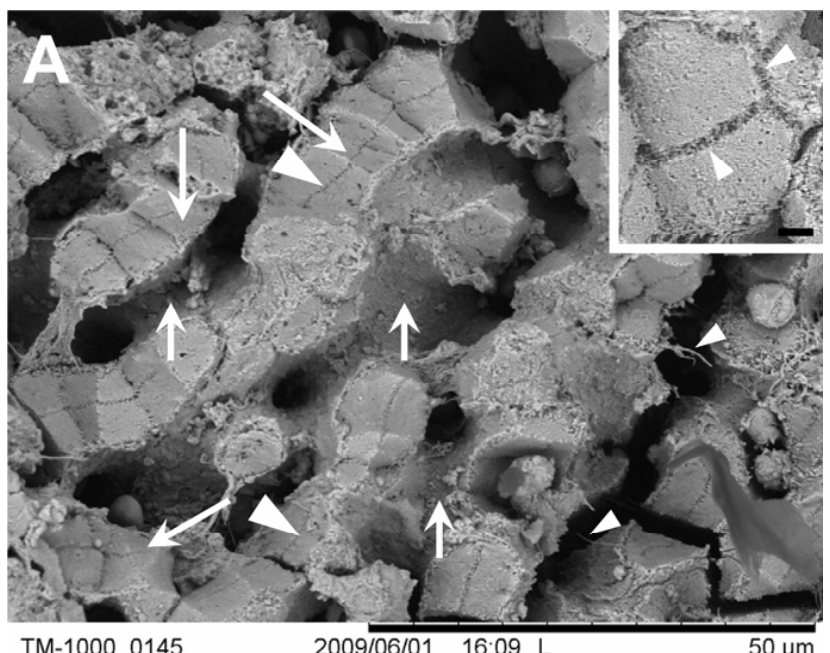

TM-1000_0145

$2009 / 06 / 01 \quad 16: 09$ L

50 um

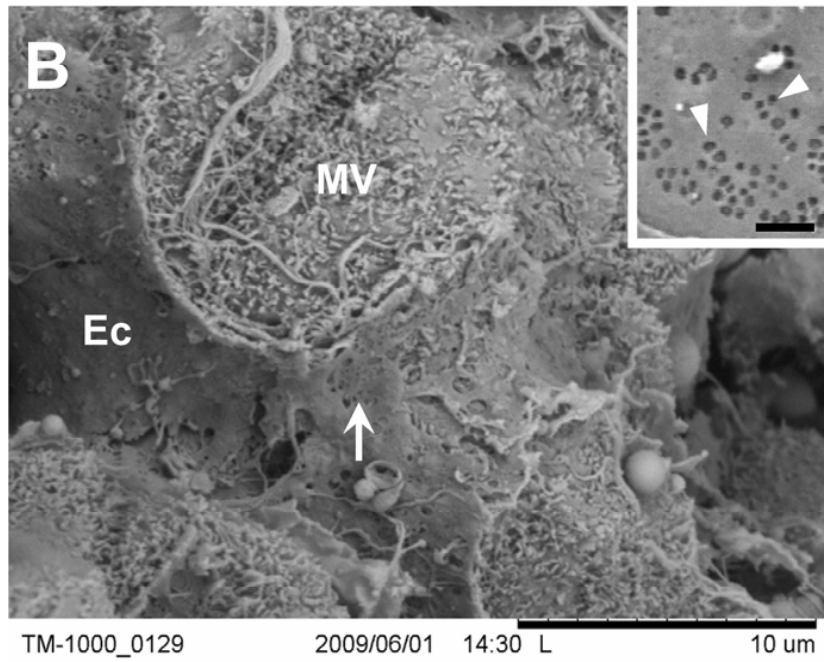

Fig. (3). The ultrastructure of liver tissue as seen at high magnification. (A) The sinusoids are readily visible (small arrows) and are surrounded by hepatocytes (large arrow). Large arrowhead, bile canaliculi; small arrowhead, extracellular matrix components. Inset depicts a detailed view of bile canaliculi (arrowheads). Scale bar, $1 \mu \mathrm{m}$. (B) Investigation of the sinusoidal lumen-which is delineated by the endothelial lining (Ec) - shows the presence of fenestrae (arrow). Note also the numerous microvilli projecting from the hepatocytes (MV). Inset shows fenestrations (arrowheads) recorded at the highest possible magnification-i.e. 10,000×-with a desktop scanning electron microscope. Scale bar, $1 \mu \mathrm{m}$.

hexamethyldisilazane has been described as a good alternative for a variety of biological samples [5, 6]. It is well known that air-drying of biological samples in water is disastrous, because of the destructive action of the surface tension during drying. Hexamethyldisilazane, however, has a reduced surface tension and also cross-links proteins, therefore adding strength to the sample during air-drying. This is supposed to reduce fracturing and collapsing of the specimen $[15,16]$. All of this is in direct support of the present observation on liver tissue whereby hexamethyldisilazane does not affect the integrity of fine structural details. 
In conclusion, a fast sample preparation method for the preservation of liver tissue for subsequent scanning electron microscopy investigation is herein presented. This method allows the facile collection of intermediate- up to high magnification electron microscopy data within an hour after the initial preparation step of liver tissue. Fine ultrastructural details within the nanometer regime can be easily retrieved from samples which are directly comparable to data obtained from samples prepared with conventional methods and imaging techniques, which requires more time and effort, including the cost of 'high-end' microscopes. This approach is therefore well-suited for implementation in basic and clinical settings in which time between experimentation and observation is central in the guidance to successive experimental testing thereby limiting the number of laboratory animals. In addition, the investigation of liver tissue after pharmacodynamic drug screening studies in animals or in the diagnostic examination of liver biopsies obtained from patients in the operating theatre would largely benefit from this approach.

Finally, it is noteworthy to flag that excellent standard sample preparation and scanning electron imaging methods do exist for the fine structure observation of liver tissue [9, 17]. These protocols have been proven successful at magnifications up to $30,000 \times$ and higher, and are without any doubt the first method of choice when the highest reachable resolution wants to achieved. This is particularly the case in field-emission scanning electron microscopy investigations that permit imaging down to one nanometer resolution.

\section{ACKNOWLEDGEMENTS}

The author is indebted is to Hitachi - Meeco Holdings Pty Ltd Australia and to Dr Joanna Biazik for reading of this manuscript.

\section{REFERENCES}

[1] Braet F, Ratinac K. Creating next-generation microscopists: structural and molecular biology at the crossroads. J Cell Mol Med 2007; 11: 759-3.
[2] Hayat MA. Principles and techniques of electron microscopy: biological applications: Cambridge University Press, USA 2000.

[3] Boyde A, Wood, C. Preparation of animal tissues for surfacescanning electron microscopy. J Microsc 1969; 90: 221-9.

[4] Boyde A. Review of basic preparation techniques for biological scanning electron microscopy. In: Brederoo P, de Priester W, editor. Proceedings of the Electron Microscopy; 1980; The Hague, The Netherlands. 768-7.

[5] Dekker NP, Lammel CJ, Brooks GF. Scanning electron microscopy of piliated Neisseria gonorrhoeae processed with hexamethyldisilazane. J Electron Microsc Tech 1991; 19: 461-7.

[6] Bray DF, Bagu J, Koegler P. Peldri II, and critical-point drying methods for scanning electron microscopy of biological specimens. Microsc Res Techn 1993; 26: 489-95.

[7] Braet F, De Zanger R, Wisse E. Drying cells for SEM, AFM and TEM by hexamethyldisilazane: A study on hepatic endothelial cells. J Microsc 1997; 186: 84-7.

[8] Horn T, Christoffersen P. Perfusion fixation of hepatic needle biopsies for scanning electron microscopy. A methodological study. Liver 1986; 6: 89-97.

[9] Wisse E, De Zanger RB, Jacobs R, McCuskey RS. Scanning electron microscope observations on the structure of portal veins, sinusoids and central veins in rat liver. Scan Electron Microsc 1983; 3: 1441-52.

[10] Wisse E, Braet F, Duimel H, et al. Assesment of fixation methods for the study of human and other livers by electron microscopy. World J Gastroenterol 2010; 16: 2851-66.

[11] Wisse E, De Wilde A, De Zanger R. Perfusion fixation of human and rat liver tissue for light and electron microscopy: a review and assessment of existing methods with special emphasis on sinusoidal cells and microcirculation. Sci Biol Spec Preparat 1984: 31-38.

[12] Frederik PM, Bomans PHH, Braet F, Wisse E. Cryo-electron microscopy of cultured hepatic endothelial cells. In: Wisse E, Knook DL, Balabaud C, Eds. Cells of the Hepatic Sinusoid 6. Leiden: Kupffer Cell Foundation, 1997: 476-8.

[13] Fahimi HD. Perfusion and immersion fixation of rat liver with glutaraldehyde. Lab Invest 1967; 16: 736-50.

[14] Wisse E. An electron microscopic study of the fenestrated endothelial lining of rat liver sinusoids. J Ultrastruct Res 1970; 31: 125-150.

[15] Nation JL. A new method using hexamethyldisilazane for preparation of soft insect tissues for scanning electron microscopy. Stain Technol 1983; 58: 347-51.

[16] Cooley WA, Scott AC, Simmons MM. The use of falcon cell culture inserts and hexamethyldisilizane for the study of small biological specimens by electron microscopy. Eur Microsc Anal 1994; 31: 17-19.

[17] Takahashi-Iwanaga H, Fujita T. Application of an $\mathrm{NaOH}$ maceration method to a scanning electron microscopic observation of Ito cells in the rat liver. Arch Histol Jpn 1986; 49: 349-57.

This is an open access article licensed under the terms of the Creative Commons Attribution Non-Commercial License (http://creativecommons.org/licenses/by-nc/ 3.0/) which permits unrestricted, non-commercial use, distribution and reproduction in any medium, provided the work is properly cited. 